\title{
The effect of a strong-probe field and transverse fields on the polarization properties of a four-level Doppler-broadened system with Spontaneously Generated Coherence (SGC)
}

\author{
Somia Abd-Elnabi \\ Department of Mathematics, Faculty of Science (Womens Section) Al-Azhar University, P.O.Box 11754, Nasr City, \\ Cairo, Egypt,
}

\section{ARTICLE INFO}

Article history:

Received 15 September 2015

Accepted 16 November 2015

\section{Keywords:}

Polarization coherence;

Doppler broadened-coherence system;

Spontaneously Generated

Coherence (SGC);

Transverse fields;

Coherent atomic system.

\section{Introduction}

The study of the strong probe field in an atomic system of a Doppler broadened in the presence of Spontaneously Generated Coherence (SGC) has been a subject of great interest in particularly when the fields are transverse mode. The transverse field is considered in a Doppler broadened molecular casade system using fluorescence detection ${ }^{[1]}$. Quantum coherence and quantum interference are the basic mechanisms for amending the optical response of the medium for the applied fields ${ }^{[2-4]}$. The intensity of a probe, coupling fields and relative phase between applied fields are the important parameters to control the atomic coherence ${ }^{[5]}$. The effects of quantum interference (SGC) in different types of atomic systems have been discussed extensively recently. A lot of theoretical work has been devoted to discuss these effects ${ }^{[6-8]}$. It has been predicted that many interesting phenomena are enhanced due to the effects of quantum interference (SGC), such as a dark resonance and the narrowing of spontaneous emission, optical bistability/multistability and modification of absorption and dispersion properties of atomic systems ${ }^{[9-11]}$. On the other hand, the phenomenon of electromagnetically induced transparency (EIT) is an alteration of probe response on a given transition when a strong control laser is applied to an auxiliary transition of a multilevel system $^{[12 f}$. In recent years, EIT in Doppler broadened media has been studied theoretically and observed exp-

\footnotetext{
* Corresponding author.

E-mail address: $\underline{\text { dr_somia_s@hotmail.com }}$
}

erimentally using cw laser sources ${ }^{[13,14]}$. Also, electromagnetically induced transparency (EIT) has been applied in a four-level N-type system for several potential applications such as high-resolution spectroscopy ${ }^{[15,16]}$. We find that in the weak-probe limit, EIT has been studied in a wide variety of fields such as lasing without inversion, polarization control and slowing of light. When EIT is applied in non-linear processes, the response to any modifications is obtained when the probe is strong. So, it is important to see the effect of a strong probe field in several applications of the phenomenon of EIT such as the splitting of the EIT resonance and narrowing absorption peak (EIA) in ${ }^{[17,18]}$. Another phenomenon is called Coherent hole burning (CHB) which could be observed in a three-level ladder and $\Lambda$-type Doppler broadened medium ${ }^{[19-24]}$. The experimental realization of large fractional pulse delays in a hot, Doppler-broadened rubidium vapor and optical control of pulse delays in cesium vapor are demonstrated and observed resulting holes in the $\mathrm{D}_{1}$ delay spectrum and also a hole is created in an inhomogeneously broadened electron spin transition and its spectrum is modified by driving hyperfine transitions ${ }^{[25-29]}$. When the perturbing laser copropagates with the probe laser, there are six (CHB) and two (EIT) that can be observed in a Doppler-broadened four level N-type atomic system by adjusting the perturbing Rabi frequency or the saturating detuning ${ }^{[30]}$. In previous reports ${ }^{[31-33]}$, we presented the Doppler broadened of the imaginary part of 
the susceptibility of probe field which represent the absorption spectrum for the four-level $\mathrm{N}$-type atomic system, we displayed a number of effects for the spectra behavior, the appearance of zero absorption points, controllability of EIT and EIA and also the spectral holes can be enhanced strongly by means of the transverse dependence of the electromagnetic fields in a Doppler broadened medium and presence of SGC. All the results are observed without including numerous hyperfine levels and Zeeman sublevels to the atomic system. The motivation of this paper, we report theoretically that the coherent holes phenomenon depends on a new condition when the probe field is strong as well as the presence of the spontaneously generating coherence (SGC) and the non radiative decay rates. We analyze the probe induced polarization spectra in a Doppler broadened four-level N-type atomic system when the transverse fields are taken into account with non-perturbative treatments of the density matrix elements solution. The paper is organized as follows: In Sec. II, Atomic model with strong probe field and transverse mode is obtained analytically. The discussion of the numerical results is given in Sec. III. Finally, a conclusion is obtained in Sec. IV.

\section{Atomic model with strong probe field and transverse mode}

The atomic system under consideration is shown in Figure 1. It can be described by a four-level N-type configuration. We consider an atom situated in transverse mode where the transverse profile of the beam's electric field distribution can be described with a Gaussian function:

$$
\exp \left(-\frac{r^{2}}{w_{i}(z)^{2}}\right)(i=1,2,3)
$$

Here, $r$ is the distance from the beam axis, $z$ is the coordinate along the propagation direction. The spot size of the Gaussian beam (the Gaussian beam radius) varies along the propagation direction according to

$$
w_{i}(z): w_{i}^{2}(z)=w_{0 i}^{2}\left(1+\frac{z^{2}}{z_{0 i}^{2}}\right)
$$

with the Rayleigh length:

$$
z_{0 i}=\frac{\pi w_{0 i}^{2}}{\lambda_{i}}
$$

and $w_{0 \mathrm{i}}$ is called the beam waist.

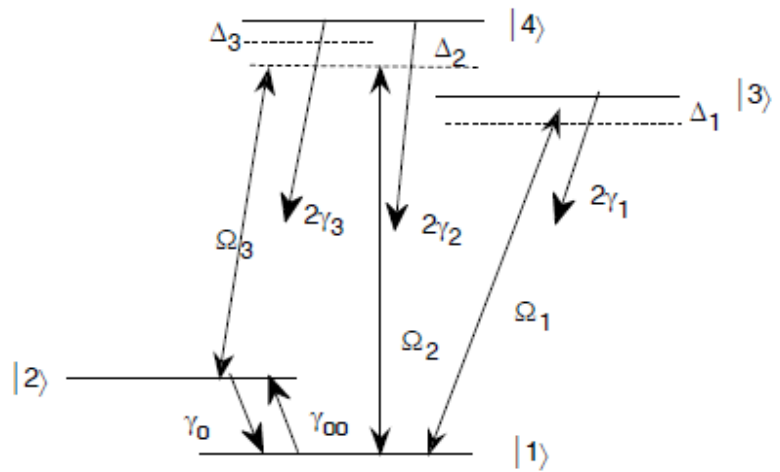

Fig 1: Schematic energy of a four-level N-type atomic system interacting with a strong probe $\left(\Omega_{2}\right)$ and pump $\left(\Omega_{1}\right)$, control $\left(\Omega_{3}\right)$ fields.
The smallest value of the spot size $w_{\mathrm{i}}(\mathrm{z})$ is at $\mathrm{z}=0$; where the spot size is equal to the beam waist parameter $w_{0 \mathrm{i}}$. In this scheme the three coherent transverse fields (pump, probe, control) have the amplitudes $E_{\mathrm{i}}(\mathrm{i}=1 ; 2$; 3 ) and the same frequencies $\omega$ interact with the transition labeled $|1\rangle \leftrightarrow|3\rangle,|1\rangle \leftrightarrow|4\rangle$ and $|2\rangle \leftrightarrow|4\rangle$, respectively. The probe field is a strong field with the transition $|1\rangle \leftrightarrow$ 47. The Rabi frequencies of the three fields are represented by the transverse profile as:

$$
\Omega_{i}(r)=\Omega_{0 i} \exp \left(-\frac{r^{2}}{w_{0 i}^{2}}\right)
$$

Where $\Omega_{01}=\left(d_{13} E_{1} / \hbar\right), \Omega_{02}=\mid\left(d_{14} E_{2} / \hbar\right)$ and $\Omega_{03}=$ $\left(d_{24} E_{3} / \hbar\right), d_{13}, d_{14}$ and $d_{24}$ are the dipolar moment of the transitions from $|i\rangle$ to $|j\rangle$. The radiative decay rates from the excited state $|4\rangle$ to the states $|1\rangle,|2\rangle$ and $|3\rangle$ to $|1\rangle$ are $2 \gamma_{2}, 2 \gamma_{3}$ and $2 \gamma_{1}$, respectively. The nonradiative decay rate from the state $|1\rangle$ to the state $|2\rangle$ is $\gamma_{00}$ and from the state $|2\rangle$ to the state $|1\rangle$ is $\gamma_{0}$. The levels $|1\rangle,|2\rangle$ and $|3\rangle$, |4) are closely lying near-degenerate, so the quantum interference (SGC), due to the spontaneous emission, can be exist. Under the rotating-wave approximation, the density-matrix equations of motion ${ }^{[34,35]}$ for the closed system take the form:

$$
\begin{aligned}
\frac{\partial \rho_{11}}{\partial t} & =i \Omega_{1}\left(\rho_{13}-\rho_{31}\right)+i \Omega_{2}\left(\rho_{14}-\rho_{41}\right)-2 \gamma_{00} \rho_{11}+2 \gamma_{0} \rho_{22} \\
& +2 \gamma_{1} \rho_{33}+2 \gamma_{2} \rho_{44}+\gamma_{12}\left(\rho_{34}+\rho_{43}\right)
\end{aligned}
$$

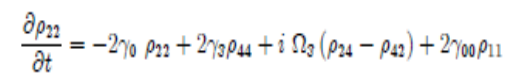

$$
\frac{\partial \rho_{33}}{\partial t}=-2 \gamma_{1} \rho_{33}-i \Omega_{1}\left(\rho_{13}-\rho_{31}\right)-\gamma_{12}\left(\rho_{34}+\rho_{43}\right)
$$

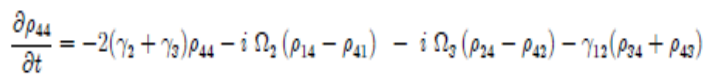

$$
\begin{gathered}
\frac{\partial \rho_{14}}{\partial t}=\left[-i \Delta_{2}-\gamma_{2}-\gamma_{3}-\gamma_{00}\right] \rho_{14}+i \Omega_{3} \rho_{12}-i \Omega_{1} \rho_{34}+i \Omega_{2}\left(\rho_{11}-\rho_{44}\right)-\gamma_{12} \rho_{13} \\
\frac{\partial \rho_{13}}{\partial t}=\left[-i \Delta_{1}-\gamma_{1}-\gamma_{00}\right] \rho_{13}-i \Omega_{2} \rho_{43}+i \Omega_{1}\left(\rho_{11}-\rho_{33}\right)-\gamma_{12} \rho_{14}
\end{gathered}
$$

$$
\begin{gathered}
\frac{\partial \rho_{12}}{\partial t}=\left[-i\left(\Delta_{2}-\Delta_{3}\right)-\gamma_{0}-\gamma_{00}\right] \rho_{12}-i \Omega_{1} \rho_{32}-i \Omega_{2} \rho_{42}+i \Omega_{3} \rho_{14}+2 \gamma_{23} \rho_{44} \\
\frac{\partial \rho_{24}}{\partial t}=\left[-i \Delta_{3}-\gamma_{2}-\gamma_{3}-\gamma_{0}\right] \rho_{24}+i \Omega_{2} \rho_{21}+i \Omega_{3}\left(\rho_{22}-\rho_{44}\right)-\gamma_{12} \rho_{23} \\
\frac{\partial \rho_{23}}{\partial t}=\left[-i\left(\Delta_{1}-\Delta_{2}+\Delta_{3}\right)-\gamma_{1}-\gamma_{0}\right] \rho_{23}+i \Omega_{1} \rho_{21}-i \Omega_{3} \rho_{43}-\gamma_{12} \rho_{24}
\end{gathered}
$$

$$
\frac{\partial \rho_{34}}{\partial t}=\left[i\left(\Delta_{1}-\Delta_{2}\right)-\gamma_{1}-\gamma_{2}-\gamma_{3}\right] \rho_{34}-i \Omega_{1} \rho_{14}+i \Omega_{2} \rho_{31}+i \Omega_{3} \rho_{32}-\gamma_{12}\left(\rho_{33}+\rho_{44}\right)
$$

the above equations are constrained by:

$$
\rho_{11}+\rho_{22}+\rho_{33}+\rho_{44}=1 \quad \text { and } \quad \rho_{i j}=\rho_{j i}^{*}
$$


The term $\gamma_{12}=p_{1} \sqrt{\gamma_{1} \gamma_{2}}$ represents the quantum interference (SGC) resulting from the cross coupling between the transitions $|1\rangle \leftrightarrow|3\rangle$ and $|1\rangle \leftrightarrow|4\rangle$. The term $\gamma_{23}=\mathrm{p}_{2} \sqrt{\gamma_{2} \gamma_{3}}$ represents the effect of SGC resulting from the cross coupling between the transitions $|1\rangle \leftrightarrow|4\rangle$ and $|2\rangle \leftrightarrow|4\rangle$. The parameters $p_{1}$ and $p_{2}$ are defined as: $p_{1}=d_{13}: d_{14} /\left|d_{13}: d_{14}\right|$ and $p_{2}=d_{14}: d_{24} / \mid$ $d_{14}: d_{24} \quad$, where the parameter $p_{1}\left(p_{2}\right)$ denotes the alignment of the two dipole moments. The existence of SGC depends on the nonorthogonality of the dipole moments $d_{14}$ and $d_{13}\left(d_{14}\right.$ and $\left.d_{24}\right)$, since it becomes maximum when $p_{1}\left(p_{2}\right)=1$ and disappear when $p_{1}\left(p_{2}\right)=$ 0 : Under consideration that one field acting on only one transition, the Rabi frequencies are modified and related to the SGC by the relations:

$$
\begin{aligned}
& \Omega_{1}(r)=\Omega_{01} \exp \left(-\frac{r^{2}}{w_{01}^{2}}\right) \sqrt{1-p_{1}^{2}} \\
& \Omega_{2}(r)=\Omega_{02} \exp \left(-\frac{r^{2}}{w_{02}^{2}}\right) \sqrt{1-p_{1}^{2}} \\
& \Omega_{3}(r)=\Omega_{03} \exp \left(-\frac{r^{2}}{n^{2}}\right) \sqrt{1-p_{2}^{2}}
\end{aligned}
$$

The Rabi frequencies $\Omega_{01}, \Omega_{02}$ and $\Omega_{03}$ correspond to zero interference and without Gaussian effect. We take $w_{01}=$ $w_{02}=w_{03}=w_{0}$. For an atom moving with velocity $v$; the coherent field frequency detunings from its atomic resonance with the same Doppler shift $k v\left(k_{1}=k_{2}=k_{3}=\right.$ $k)$ are given by: $(i=1,2,3)$

$$
\Delta_{i}=\Delta_{0 i}-k v
$$

where: $\Delta_{01}=\omega_{13}-\omega, \Delta_{02}=\omega_{14}-\omega$ and $\Delta_{03}=\omega_{24}-\omega$, denoted the detunings for the atom at rest. $\omega_{\mathrm{ij}}$ is the transition frequency from level $i$ and $j$. The driving fields are propagating in the same direction as the probe field. Then the density matrix equations of motion (2 12) can be solved at the steady state by exact analytical calculations to obtain the coherent term $\rho_{14}$ under the effect of Doppler broadening. As is known, the response of the atomic medium to the probe field is governed by its atomic polarization: $P=\epsilon_{\mathrm{o}} \chi E_{2}$, where $\chi$ being the complex susceptibility of the atomic medium, and $\epsilon_{\mathrm{o}}$ is the permittivity of free space. The induced atomic polarization is related to the density matrix element by: $P=N_{0} d_{14} \rho_{14}$ where $N_{0}$ is the atom number density of the medium. The atomic polarization is to be averaged over the Maxwellian distribution for the atomic velocities and the transverse field profiles and is given by:

$$
\left\langle P>=\frac{N_{0} d_{14}}{\frac{w_{0}^{2}}{4}\left(1-\exp \left(-\frac{2 r_{0}^{2}}{w_{0}^{2}}\right)\right) \sqrt{\pi u^{2}}} \int_{0}^{r_{0}} \exp \left(-\frac{r^{2}}{w_{0}^{2}}\right) r d r \int_{-\infty}^{\infty} \rho_{14}(r, v) \exp \left[-\frac{v^{2}}{u^{2}}\right] d v\right.
$$

Where $u=\sqrt{2 K T / m}$ represents the most probable atomic velocity, $m$ being the mass of an atom, $K$ is Boltzmann's constant, $T$ is the absolute temperature and $r_{\mathrm{o}}$ is the radial position with respect to the common axis of the beam.
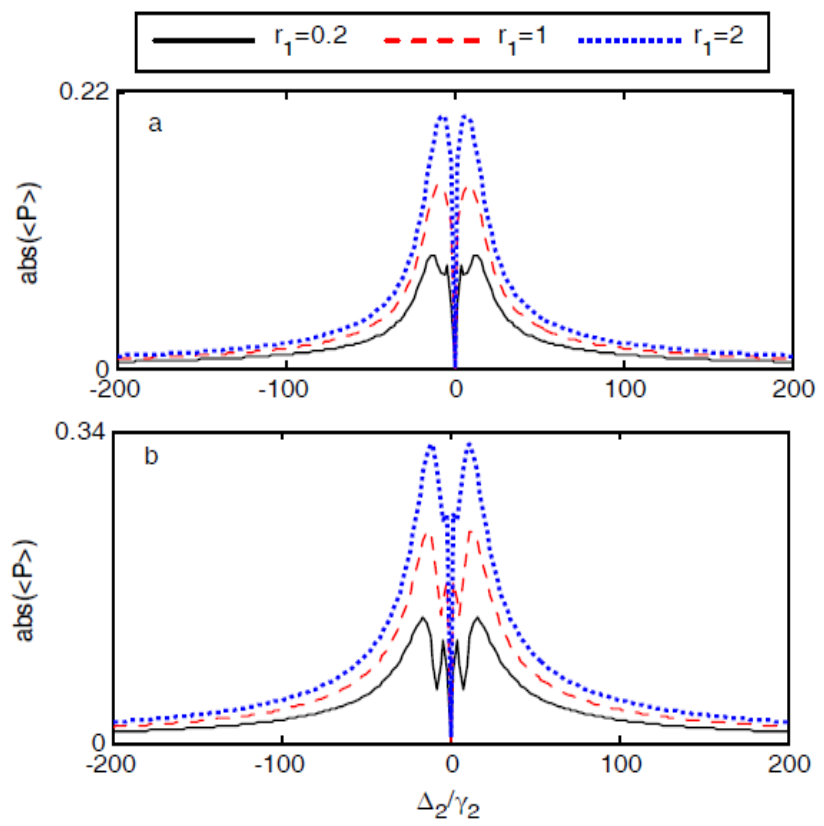

Fig 2: The probe polarization spectrum $(\operatorname{abs}(\langle\mathrm{P}\rangle))$ versus the probe frequency detuning $\left(\Delta_{2} / \gamma_{2}\right)$, for $\gamma_{1}=\gamma_{3}=\gamma_{2}$, $\Omega_{01}=4 \gamma_{2}, \Omega_{03}=7 \gamma_{2}, \mathrm{D} / \gamma_{2}=\mathrm{ku} / \gamma_{2}=10, \gamma_{00}=\gamma_{0}=0.01, \quad\left(\mathrm{p}_{1}=0.99\right.$, $\mathrm{p}_{2}=0$ ). Plot (a) for $\Omega_{02}=10 \gamma_{2}$. Plot (b) for $\Omega_{02}=20 \gamma_{2}$, at $\mathrm{r}_{1}=0.2,1,2$.

\section{Numerical Results}

We now discuss the results for the strong probe spectra of a Doppler broadened four-level $N$-type atomic system via transverse fields in the presence of SGC by performing the numerical evaluations of the integrals in Eqn. (18). The strong probe and transverse field can significantly modify the optical properties of this atomic system. We focus on the dependence of the polarization of the strong probe field on the SGC to achieve coherent hole phenomenon without saturation field. We assume the parameters $\gamma_{1}=\gamma_{3}=\gamma_{2}, \Delta_{01}=\Delta_{03}=0$ and all Figures are plotted in the unit of $\gamma_{2}$. The nonradiative decay rate from the state $|1\rangle$ to the state $|2\rangle\left(\gamma_{00}\right)$ is equal to that from the state $|2\rangle$ to the state $|1\rangle\left(\gamma_{0}\right)$, so $\left(\gamma_{0}=\gamma_{00}=0.01\right.$ $\left.\gamma_{2}\right)$, and the Doppler width is $\left(D / \gamma_{2}=k u / \gamma_{2}=10\right)$ unless another stated. The parameter $r_{1}\left(=\frac{r o}{w o}\right)$ measures the transverse effect. The two-dimensional polarization spectrum is taken as a function of the probe detuning $\left(\Delta_{2}\right)$ in the Figures.

In Figure 2, we present the behavior of the polarization spectrum under the influence of the parameter of the transverse field. The parameters of SGC are taken as $\left(p_{1}=0.99, p_{2}=0\right)$ and $\Omega_{01}=4 \gamma_{2}, \Omega_{03}=7 \gamma_{2}$. In each plot, we have three curves for different values of the parameter $r_{1}(0.2,1,2)$ to measure the transverse effect. The solid curve for $r_{1}=0.2$, the dashed curve for $r_{1}=1$ and the dotted curve for $r_{1}=2$. We now investigate the effect of a strong probe field on the polarization spectrum. At first when the Rabi frequency of strong probe field is equal $\Omega_{02}=10 \gamma_{2}$ as in Plot (a), the solid curve $\left(r_{1}=0.2\right)$ shows EIT window with two holes around the resonance and 
for $r_{1}=1$, the two holes become small as in dashed curve, while the dotted curve has an EIT window only, when increasing the transverse field parameter at $\left(r_{1}=\right.$ 2 ). The coherent holes are observed obviously when the Rabi frequency of strong probe field has an intensity: $\left(\Omega_{02}=20 \gamma_{2}\right)$ as in Plot $(b)$, the two holes have great depth at $r_{1}=0.2$ (solid curve). The depth of the holes is decreased and the external wings of the holes ascend to the higher values when $r_{1}=1$. The polarization spectrum shows two holes which fall in the EIT window with increasing the transverse field parameter $\left(r_{1}=2\right)$ in the dotted curve. The polarization spectra are modified for each value of the transverse field parameter, so we can control the quality of the coherent hole via the transverse field parameter.

Now, we investigate the effect of the spontaneously generated coherence (SGC) on the polarization spectrum in Figure 3, plots (a), (b) and (c) are used for $p_{2}=0.2$, $0.6,0.8$, respectively and $p_{1}=0.99$ and plots $(\mathrm{d}),(\mathrm{e})$ and (f) for $p_{1}=0.97,0.9,0.6$, respectively and $p_{2}=0$. The parameter of the transverse field is taken as $r_{1}=0.2$, the intensity of the Rabi frequency of probe fields are taken as: $\Omega_{02}=20 \gamma_{2}$ and the other data as in Figure 2. We observe from plots (a), (b) and (c) that the coherent holes disappear as the parameter of the (SGC) $p_{2}$ is increased (which results from the cross coupling the levels $|1\rangle,|2\rangle$ with level $|4\rangle)$ where we obtain EIT window only in plot (c). Unlike the plots (d), (e) and (f) which show that holes disappear as the parameter of the (SGC) $p_{1}$ are decreased (which result from the cross coupling the levels $|3\rangle,|4\rangle$ with level $|1\rangle)$. The striking point, we observed that the coherent holes are inside EIT window which has became wide (narrowness) when the parameter of (SGC) $p_{1}\left(p_{2}\right)$ decreased (increased).
We discuss the influence of the parameter of the Doppler width $(D)$ on the polarization spectrum in Figure 4. The parameters of SGC are taken as $\left(p_{1}=0: 99, p_{2}=0\right)$ in plot (a), $\left(p_{1}=0.9, p_{2}=3\right)$ in plot $(\mathrm{b}),\left(p_{1}=0.9, p_{2}=0.9\right)$ in plot (c) and the other data as in Figure 3. We plot the two-dimensional polarization spectrum with different values for $(D=5,20,40)$, the solid curve is considered for $D=5$, the dashed curve for $D=20$ and the dotted curve for $D=40$. At $D=5$, we have three cases in plots (a), (b), (c): for $\left(p_{1}=0.99, p_{2}=0\right)$ as in plot (a), the polarization spectrum show two deep holes on the two sides of the EIT window. In plot (b), for $\left(p_{1}=0.9, p_{2}=3\right)$ the deep of the holes are reduced, but at $\left(p_{1}=0.9, p_{2}=\right.$ 0.9 ) as in plot (c), the holes disappear and we have only EIT window. We notice also that at the parameter of the Doppler width $(D=20 ; 40)$, the coherent holes became broad and wide as well as the polarization spectrum is suppressed.

Figure 5 exhibits the influence of coherent pumping and coupling fields on the probe polarization spectrum for various intensity of the pumping field with Rabi frequency $\left(\Omega_{01}=6 \gamma_{2}, 26 \gamma_{2}, 36 \gamma_{2}\right)$ as shown in plots (a), (b) and (c), respectively and for various intensity of the Rabi frequency of coupling field $\left(\Omega_{03}=4 \gamma_{2}, 24 \gamma_{2}, 34 \gamma_{2}\right)$ which displayed in plots (d), (e) and (f), respectively. The two parameters of SGC are taken as $\left(p_{1}=0.99, p_{2}=\right.$ 0 ) and the other data as in Figure 3. When we increase the intensity of the Rabi frequency of pumping field $\left(\Omega_{03}\right)$, the coherent holes fall inside EIT window gradually until they disappear as in plots (a), (b) and (c). But when we increase the intensity of the Rabi frequency of coupling field $\left(\Omega_{03}\right)$, the coherent holes rise to the top of the polarization spectrum outside the EIT window as well as increasing the width of the EIT window.
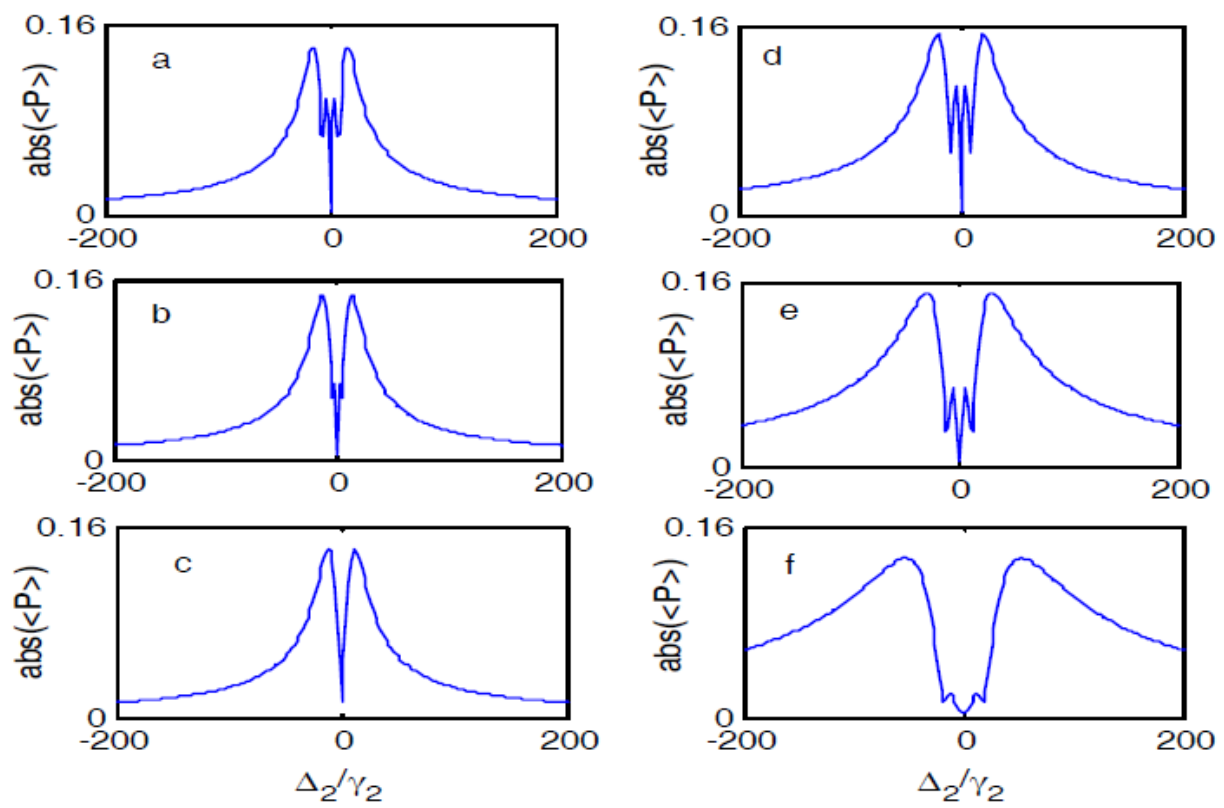

Fig 3: The probe polarization spectrum $(\operatorname{abs}(<\mathrm{P}\rangle))$ versus the probe frequency detuning $\left(\Delta_{2} / \gamma_{2}\right)$, for $\Omega_{02}=20 \gamma_{2}, \mathrm{r}_{1}=0.2$, and other data as in Figure 2. Plots (a), (b), (c) for $\left(\mathrm{p}_{1}=0.99, \mathrm{p}_{2}=0.2,0.6,0.8\right)$. Plots (d), (e), (f) for $\left(\mathrm{p}_{2}=0, \mathrm{p}_{1}=0.97,0.9,0.6\right)$. 


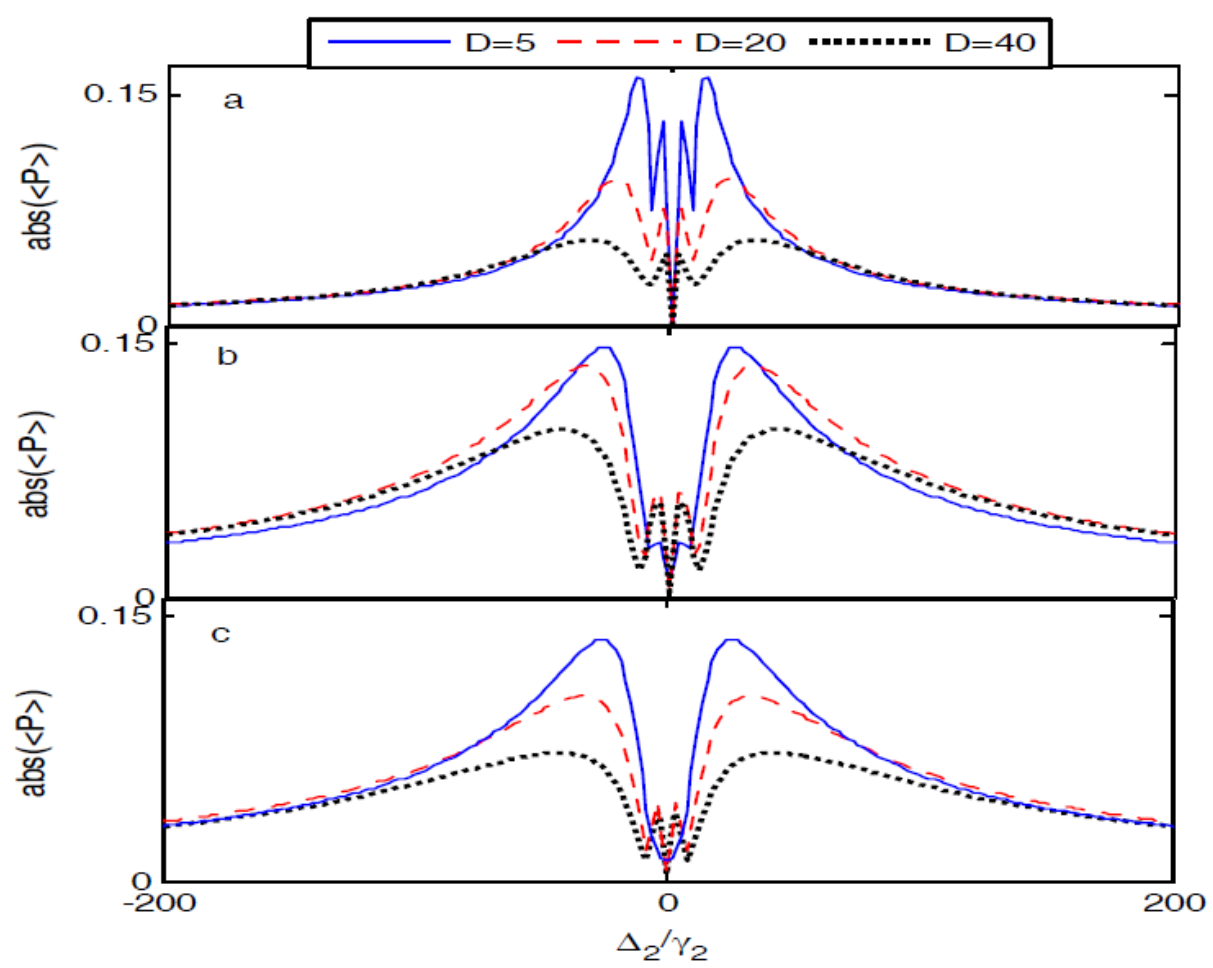

Fig 4: The probe polarization spectrum $(\operatorname{abs}(<\mathrm{P}>))$ versus the probe frequency detuning $\left(\Delta_{2} / \gamma_{2}\right),\left(p_{1}=0.99, p_{2}=0\right)$ in plot (a), $\left(p_{1}=0.9, p_{2}=0.3\right)$ in plot (b), $\left(p_{1}=0.9, p_{2}=0.9\right)$ in plot $(\mathrm{c})$, for $(D=5,20,40)$. The other data are as in Figure 3.
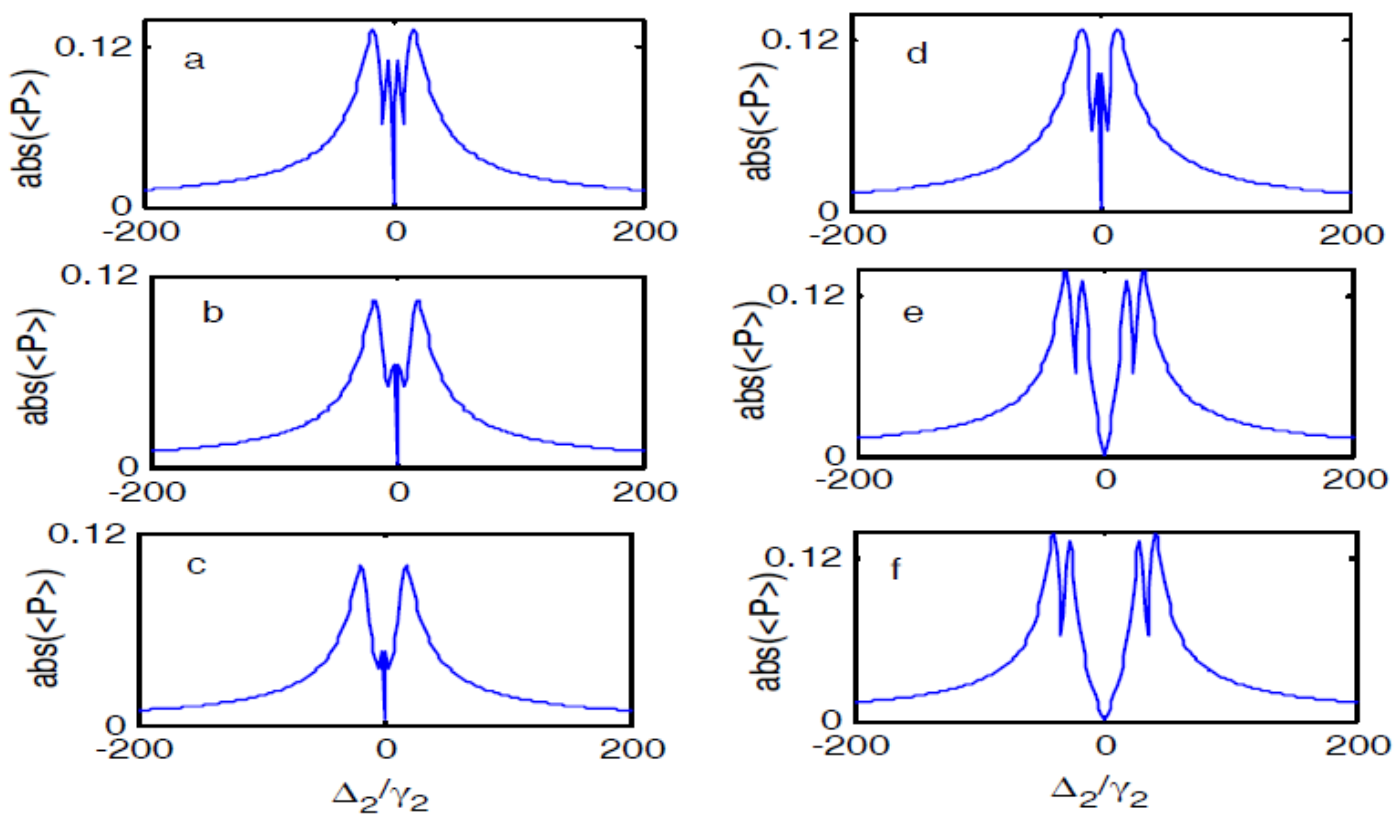

Fig 5: The probe polarization spectrum $(\operatorname{abs}(<\mathrm{P}\rangle))$ versus the probe frequency detuning $\left(\Delta_{2} / \gamma_{2}\right)$, for $\left(\Omega_{01}=6 \gamma_{2}, 26 \gamma_{2}, 36 \gamma_{2}\right)$ in plots (a), (b) and (c). $\left(\Omega_{03}=4 \gamma_{2}, 24 \gamma_{2}, 34 \gamma_{2}\right)$ in plots (d), (e) and (f) for $\left(p_{1}=0.99, p_{2}=0\right)$. The other data are as in Figure 3.

The nonradiative decay rates are considered the main source of the coherent holes, we examine the effect of the nonradiative decay rates on the polarization spectrum in Figure 6. The Doppler width $(D=10)$ and the other data are as in Figure 4. In plot $(a)$, enlarged spectrum is observed at small value of $\gamma_{0}(=0.04)$ for the dotted curve, and we also obtain two deep coherent holes. When increasing the nonradiative decay rates at $\left(\gamma_{0}=0.6\right)$ in the solid curve, the depth of the coherent holes is reduced. At $\left(\gamma_{00}=1.6\right)$, the two holes are dropped into the EIT window which are exhibited only as shown in the dashed curve. In plot $(b)$, we present plot $(a)$ in the three-dimensions to show the dependence and behavior of the probe polarization spectrum explicitly on the nonradiative decay rate. 

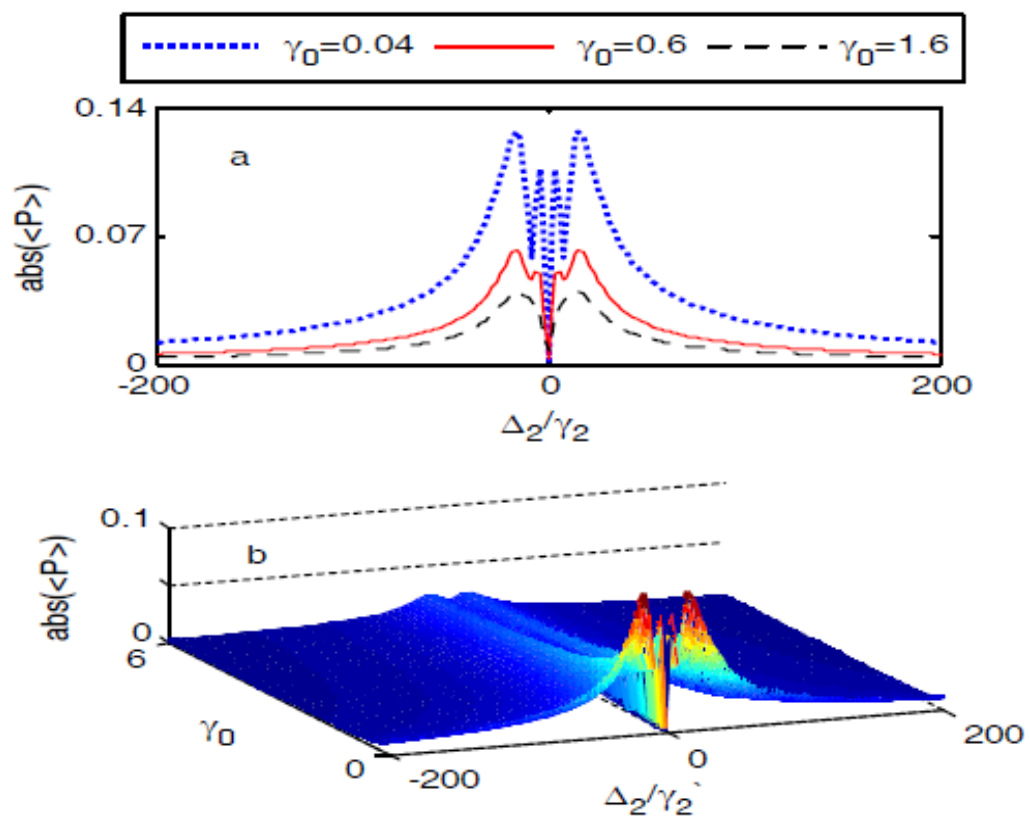

Fig 6: In plot (a), the probe polarization spectrum $(\operatorname{abs}(<\mathrm{P}\rangle))$ versus the probe frequency detuning $\left(\Delta_{2} / \gamma_{2}\right)$, for $\left(\gamma_{0}=0.04,0.6,1.6\right)$. Plot (b) for the 3D probe polarization spectrum $(\operatorname{abs}(\langle\mathrm{P}\rangle))$ as a function of the nonradiative decay rate $\left(\gamma_{0}\right)$ and the probe frequency detuning $\left(\Delta_{2} / \gamma_{2}\right)$, and other data as in Figure 4.

\section{Conclusion}

We have studied theoretically the probe polarization spectra of a Doppler broadened four-level $N$ - type atomic system driven with the transverse fields. The strong probe field has been used to produce the coherent holes which appear deeper and clearer in the probe polarization spectrum when we increase the strong probe field. The coherent holes become much more controllable in width and depth by the transverse field parameter. The parameters of the spontaneously generated coherence (SGC) are important in displaying the hole phenomenon. The nonradiative decay rates have a major role in the appearance the coherent holes. By adjusting the intensity of the Rabi frequency of pumping field $\left(\Omega_{01}\right)$ or the intensity of the Rabi frequency of coupling field $\left(\Omega_{03}\right)$; we can observe different behaviors of the coherent holes. The holes phenomenon can be observed clearly by the $3 D$ polarization spectrum when the probe polarization spectrum depends upon the nonradiative decay rate parameters. Finally, the properties of the probe polarization spectra of a Doppler broadened four-level $N$ - type atomic system as well as the coherent holes phenomenon are more significantly modified by the strong probe field and the transverse parameter.

\section{References}

1) Qi, J. et al. (2002). Measurement of Transition Dipole Moments in Lithium Dimers Using Electromagnetically Induced Transparency. Phys. Rev. Lett., 88: 173003.

2) Agarwal, G. S. (1974). In Quantum Optics, Springer Tracts in Modern Physics, vol. 70, Springer Berlin/ Heidelberg.
3) Whitley, R. M. and Stroud Jr., C. R. (1976). Double optical resonance. Phys. Rev. A, 14: 1498.

4) Osman, K. I. and Abd-Elnabi, S. (1991). The effects of laser cross-correlation in a four-level system. Opt. Communications, 83: 49.

5) Agarwal, G. S., Dey, T. N. and Menon, S. (2001). Knob for changing light propagation from subluminal to superluminal. Phys. Rev. A, 64: 053809.

6) Zhu, S. Y., Chan, R. C. F. and Lee, C. P. (1995). Spontaneous emission from a three-level atom. Phys. Rev. A, 52: 710.

7) Zhou, P. and Swain, S. (1997). Quantum Interference in Probe Absorption: Narrow Resonances, Transparency, and Gain without Population Inversion. Phys. Rev. Lett., 78: 832.

8) Osman, K. I., Hassan, S. S. and Joshi, A. (2009). Effect of spontaneously generated coherence on EIT and its refractive properties in four- and five-level systems. European Physical Journal D, 54: 119.

9) Liu, C. P., Gong, S. Q., Fan, X. J. and Xu, Z. Z. (2004). Electromagnetically induced absorption via spontaneously generated coherence of a $\Lambda$ system. Opt. Communications, 231: 289.

10) Osman, K. I. (2006). Effects of the control field on the optical multistability in V-type three-level atomic system. Opt. Communications 259: 194.

11) Osman, K. I. and Joshi, A. (2013). Induced coherence and optical bistability in a four-level system with incoherent pumping. Opt. Communications, 293: 86.

12) Harris, S. E. (1997). Electromagnetically induced transparency. Phys. Today, 50: 36. 
13) Xiao, M., Li, Y. Q., Jin, S. Z. and Banacloche, J. G. (1995). Measurement of dispersive properties of electromagnetically induced transparency in rubidium atoms. Phys. Rev. Lett., 74: 666.

14) Lee, H., Rostovtev, Y., Bednar, C. J. and Javan, A. (2003). From Laser Induced Line Narrowing To Electromagnetically Induced Transparency: Closed System Analysis. Appl. Phys. B, 76: 33.

15) Akulshin, A. M., Barreiro, S. and Lezama, A. (1998). Electromagnetically induced absorption and transparency due to resonant two-field excitation of quasidegenerate levels in $\mathrm{Rb}$ vapor. Phys. Rev. A, 57: 2996.

16) Kong, L. B., Tu, X. H., Wang, Zhu, J. Y. and Zhan, M. S. (2007). Sub-Doppler spectral resolution in a resonantly driven four-level coherent medium. Opt. Communications, 269: 362.

17) Pandey, K. and Natarajan, V. (2008). Splitting of electromagnetically induced transparency under strong-probe conditions due to Doppler averaging. J. phys. B: At. Mol. Opt. phys., 41: 185504.

18) Pandey, K., Kaundilya, D. and Natarajan, V. (2011). Role of population transfer under strong probe conditions in electromagnetically induced transparency. Opt. Communications, 284: 252.

19) Dong, P. and Gao, J. Y. (2000). Appearance and disappearance of hole-burning behind an electromagnetically induced transparency window. Phys. Lett. A, 265: 52.

20) Xiao, Z. H., Wu, J. H., Zhang, H. F. and Gao, J. Y. (2003). Comparison of coherent induced holeburning between $\Lambda, \mathrm{V}$ and ladder systems. Phys. Lett. A, 310: 363.

21) Wu, J. H., Wei, X. G., Wang, D. F., Chen, Y. and Gao, J. Y. (2004). Coherent hole-burning phenomenon in a Doppler broadened three-level $\Lambda$ type atomic system. J. Opt. B, 6: 54.

22) Perettia, R., Jurdyca, A. M., Jacquiera, B., Burovb, E. and Gascab, L. (2008). Resonant fluorescence line narrowing and gain spectral hole burning in erbium-doped fiber amplifier. $\mathrm{J}$. Luminescence, 128: 1010.

23) Kuang, S. Q., Du, P., Wan, R. G., Jiang, Y. and Gao, J. Y. (2008). Slow light based on coherent hole-burning in a Doppler broadened three-level $\Lambda$ type atomic system. Optics Express 16: 11604.
24) He, Q. Y., Wei, X. G., Wu, J. H., Zhang, B. and Gao, J. Y. (2008). Coherent hole-burnings induced by a bichromatic laser field. Opt. Communications, 281: 3137.

25) Wei, X. G., Wu, J. H., Wang, H. H., Li, A., Kang, Z. H., Jiang, Y. and Gao, J. Y. (2006). Firstprinciples experimental observation of coherent hole burnings in atomic rubidium vapor. Phys. Rev. A, 74: 063820 .

26) Agarwal, G. S. and Dey, T. N. (2003). Slow light in Doppler-broadened two-level systems. Phys. Rev. A, 68: 063816.

27) Camacho, R. M., Pack, M. V. and Howell, J. C. (2006). Slow light with large fractional delays by spectral hole-burning in rubidium vapor. Phys. Rev. A, 74: 033801.

28) Anderson, M. D. and Perram, G. P. (2012). Optical delay with spectral hole burning in Dopplerbroadened cesium vapor. Opt. Communications, 285: 3264.

29) Manson, N. B., Rogers, L. J., Wilson, E. A. and Wei, C. (2010). Hole burning-EIT studies of the NV centre in diamond. J. Luminescence, 130: 1659.

30) Tian, X. X., Li, D. K. and Wu, J. H. (2010). Coherently induced hole-burnings in a Doppler broadened four-level atomic system. Opt. Communications, 283: 2561.

31) Abd-Elnabi, S. and Osman, K. I. (2013). Controllability of electromagnetically induced transparency in a Doppler broadened four-level atomic system. International Journal of Modern Physics B, 27: 1350037.

32) Abd-Elnabi, S. (2014). Coherent hole burnings induced without saturation field in a Doppler broaden four-level N-type atomic system. Optik-International Journal for Light and Electron Optics, 125: 16201624.

33) Abd-Elnabi, S. and Osman, K. I. (2014). Gaussian field induced spectral hole burning in a Doppler broadened system and spontaneously generated coherence. Journal of Luminescence, 147: 346.

34) Scully, M. O. and Zubairy, M. S. (1997). Quantum Optics Cambridge University Press Cambridge.

35) Meystre, P. and Sergent, M. (1999). Elements of Quantum Optics, $3^{\text {rd }}$ ed. springer-verlag, Berlin. 